\title{
Das Praxisassistenzprogramm des Instituts für Hausarztmedizin am UniversitätsSpital Zürich
}

\author{
Ziel der sechsmonatigen Praxisassistenz ist eine qualitativ hochwertige Weiterbil- \\ dung, die umfassenden Einblick in die Vielfalt und Komplexität der hausärztlichen \\ Tätigkeit ermöglicht. Das Angebot richtet sich bevorzugt an Bewerber, die in der \\ Facharztweiterbildung bereits fortgeschritten sind.
}

\section{Thomas Rosemann ${ }^{a}$, Ryan Tandjung ${ }^{b}$, \\ Elisabeth Bandi-Ottc \\ a Prof. Dr. med., PhD, Direktor Institut für Hausarztmedizin Zürich \\ b Dr. med., Oberarzt, USZ \\ c KD Dr. Elisabeth Bandi-Ott, USZ, Leitung Lehre}

* Es sind selbstverständlich immer beide Geschlechter gemeint.

Korrespondenz:

Prof. Dr. med.

Thomas Rosemann, PhD

Institut für Hausarztmedizin

UniversitätsSpital Zürich

Pestalozzistrasse 24

CH-8091 Zürich

thomas.rosemann[at]usz.ch
Der Nachwuchsmangel in der Hausarztmedizin ist evident und stellt eine Bedrohung für die qualitativ hochwertige und zugleich kostengünstige Versorgung der Schweizer Bevölkerung dar. Das Institut für Hausarztmedizin der Universität Zürich (IHAMZ) versucht, dem auf allen Ebenen der medizinischen Aus-, Weiter- und Fortbildung entgegenzuwirken. Neben zahlreichen Vorlesungen und Kursen im Studium bieten Masterarbeiten und Dissertationen die Möglichkeit zum intensiven Kontakt mit der Hausarztmedizin als medizinischer Spezialität. Studien zeigen jedoch, dass die Wahl der späteren medizinischen Fachspezialität durch die jungen Ärzte ganz wesentlich vom Angebot an qualitativ hochwertigen und adäquat entlohnten Weiterbildungsstellen bestimmt wird [1]. Das IHAMZ bietet daher mit Unterstützung des UniversitätsSpitals Zürich (USZ) seit drei Jahren ein spezielles Hausarzt-Curriculum an, das sich grosser Nachfrage erfreut und damit eindrucksvoll untermauert, dass die Hausarztmedizin durchaus attraktiv ist, wenn es adäquate Weiterbildungsangebote gibt [2]. Aufgrund der grossen Nachfrage und der positiven Erfahrungen mit dem Hausarzt-Curriculum wurde nun zusätzlich zum 1. Januar 2013 am USZ ein Praxisassistenzprogramm lanciert. Es ist als Alternative zu etablierten Angeboten wie dem der Stiftung WHM-FMF (www.whm-fmf.ch) oder zu Angeboten einzelner Spitäler zu sehen.

\section{Ziel und Inhalt}

Das Ziel der sechsmonatigen Praxisassistenz ist eine qualitativ hochwertige Weiterbildung in der Hausarztmedizin, die einen umfassenden Einblick in die Vielfalt und Komplexität der hausärztlichen Tätigkeit ermöglicht. Das Angebot der Praxisassistenz richtet sich bevorzugt an Bewerber*, die in der Facharztweiterbildung bereits fortgeschritten sind und sich mittels der Praxisassistenz auf die Tätigkeit als Grundversorger vorbereiten möchten. Es steht aber auch Kollegen offen, die sich hinsichtlich ihrer späteren Tätigkeit noch nicht sicher sind und sich über eine Tätigkeit in einer hausärztlichen Praxis orientieren wollen.

\section{Regelmässige Fortbildungen als wesentlicher Bestandteil}

Das Praxisassistenzprogramm des IHAMZ (IHAMZPA) unterscheidet sich in mancherlei Hinsicht von den bekannten Programmen. Während die etablierten Programme in der Regel rein passive CoFinanzierungsprogramme sind, die dazu dienen, den finanziellen Aufwand des aufnehmenden Lehrarztes abzumildern, ist das IHAMZ Programm als aktives Weiterbildungsprogramm zu verstehen. Ein zentrales Element der Praxisassistenz sind regelmässige Fortbildungsveranstaltungen, die vom IHAMZ organisiert werden. Im Mittelpunkt stehen dabei klinisch-praktische Themen, die häufig im hausärztlichen Alltag vorkommen. Da das Institut keine eigene Ambulanz hat, wird dazu die institutsassoziierte Praxis genutzt. Neben den Institutsmitarbeitern stehen dort auch Spezialisten aus dem USZ als kompetente Referenten zur Verfügung. Praktisch orientierte interaktive Übungen zu kardiologischen, angiologischen, pulmonologischen, dermatologischen, rheumatologischen und psychiatrischen Themen runden die Fortbildungen ab. Zudem werden auch allgemeine Themen rund um die Niederlassung aufgegriffen, wie etwa «Praxissuche», «Finanzierung», «elektronische Krankengeschichte» oder auch «standespolitische Einrichtungen und ihre Aufgaben». Wichtig dabei ist es, objektive und unabhängige Informationen zu geben, kommerzielle und interessensgeleitete Anbieter wie in anderen «Existenzgründungsseminaren» haben keinen Zugang.

\section{Vernetzung und Austausch}

Viele junge Kollegen sind auf dem Weg in die (eigene) Praxis alleingelassen und haben nur wenig Möglichkeit zum Austausch unter ihresgleichen. Zudem besteht oft eine gewisse Zurückhaltung, über Unsicherheiten und finanzielle Aspekte im Zusammenhang mit der Existenzgründung oder auch der Tätigkeit im Angestelltenverhältnis in der ambulanten Versorgung zu sprechen. Die ausführlichen Apéros nach jeder Fortbildung schaffen hierfür den entspannten Rahmen. Um den Kreis über die jeweiligen Praxis- 
und Curriculumsassistenten zu erweitern, sind auch die Jungen Hausärzte Schweiz sowie frühere Absolventen des Programmes eingeladen, ihre Erfahrungen weiterzugeben. Zu ausgewählten Veranstaltungen werden auch Studierende höherer Semester eingeladen. So spannt sich ein Bogen von den Studierenden über die Kollegen im Curriculum und der Praxisassistenz bis hin zu den frischgebackenen Hausärzten und es ergeben sich regelmässig angeregte Diskussionen.

\section{Regelmässiges Mentoring}

Am IHAMZ wurde für die Organisation, vor allem aber auch für das Mentoring der Praxisassistenten eine Oberarztposition geschaffen. Damit steht ein Ansprechpartner für alle Sorgen und Nöte während

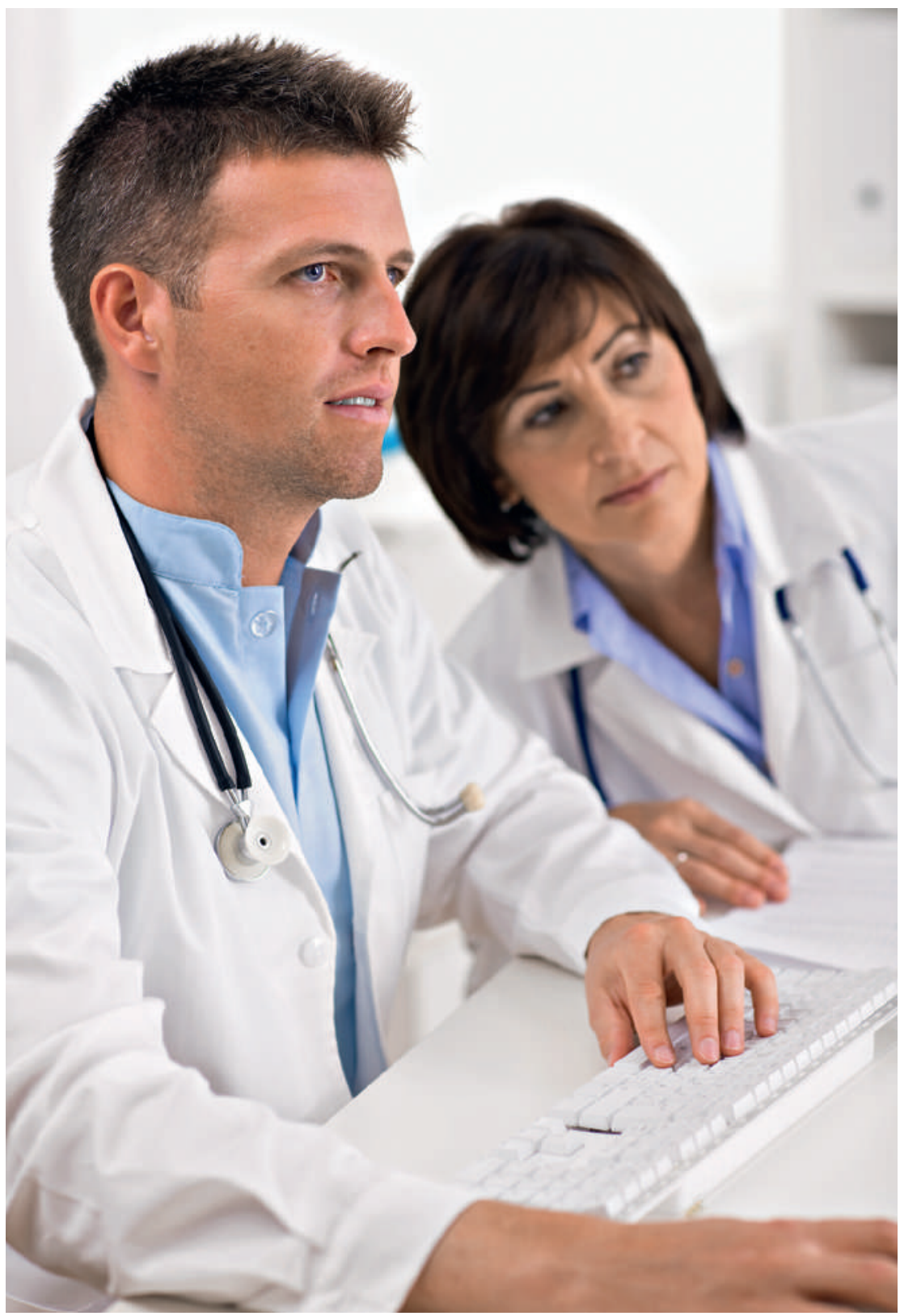

Während der sechsmonatigen Praxisassistenz können sich zukünftige Hausärzte bei einem Lehrpraktiker auf die Tätigkeit als Grundversorger vorbereiten. der Praxisassistenz zur Verfügung. Auch eine Visitation aller Weiterbildungspraxen für deren Akkreditierung sichert die Qualität der Weiterbildung. Eine weitere wichtige Aufgabe ist die Auswertung der regelmässigen Evaluationen der Praxen durch die Assistenten.

\section{Finanzierung und Entlohnung}

Der höhere Aufwand für Assistenten ist andererseits mit einigen positiven finanziellen Aspekten assoziiert. Die Assistenten werden über das UniversitätsSpital Zürich angestellt und gemäss den Richtlinien der Einstufung (gemäss HRM USZ) wie im Spital tätige Kollegen mit gleicher Qualifikation entlohnt. Das bezahlte Salär bezieht sich somit auch auf ein 50-Stunden-Pensum. Sämtliche Lohnnebenkosten und Sozialbeiträge werden durch das USZ geleistet. Damit ist erstmals gewährleistet, dass die Praxisassistenz keine finanziellen Nachteile für den hausärztlichen Nachwuchs mit sich bringt.

Abhängig von der Einstufung der Assistenzärzte bezahlt der Lehrpraktiker einen monatlichen Fixbetrag, der je nach Qualifikation des Assistenten zwischen 3000 Franken und 3500 Franken variiert, an die Kostenstelle des IHAMZ am USZ zurück. Ansonsten fällt kein administrativer Aufwand für den Lehrpraktiker an, einzig die Haftpflichtversicherung für den Praxisassistenten fällt in seinen Verantwortungsbereich.

Das Praxisassistenzprogramm kann in einem 100\%-, 50\%- und 80\%-Pensum absolviert werden. Das parallel angebotene 2-jährige Curriculum, das im letzten Abschnitt ebenfalls eine sechsmonatige Praxisassistenz beinhaltet, kann aus organisatorischen Gründen nur in einem 100\%-Pensum absolviert werden. Insgesamt stehen 15 Stellen zur Verfügung, da jede Praxisassistenz 6 Monate dauert, resultieren daraus 30 Praxisassistenzen pro Jahr.

\section{Anforderungen an interessierte Assistenzärzte}

Assistenten, die sich für das PA-Programm am IHAMZ interessieren, bewerben sich mit den üblichen Unterlagen, inkl. Lebenslauf und Diplomen, direkt beim Direktor des IHAMZ. Eine Bewerbung per E-Mail mit vollständigen elektronischen Unterlagen wird gewünscht und sollte so früh wie möglich vor der geplanten Assistenz erfolgen. Die Bewerbung sollte eine Erklärung zur Motivation für den Eintritt in das jeweilige Programm (Praxisassistenz oder Curriculum) enthalten. Ganz wichtig und ein entscheidender Unterschied zum WHM Programm ist dabei, dass die Zuteilung auf die Praxen durch das Institut erfolgt. «Tandem»-Bewerbungen von Lehrpraktiker und Assistent sind daher nicht aussichtsreich. Das Institut stellt den Assistenten eine Liste aktueller, akkreditierter Lehrpraktiker zur Verfügung, die die Kriterien des Institutes an Weiterbildungsqualität und Zusammenarbeit erfüllen. Natürlich werden - so weit möglich - 


\section{Ein wichtiges Anliegen der Praxisassistenz ist auch die Vernetzung und der Austausch unter den zukünftigen Hausärzten.}

Wünsche im Hinblick auf die räumliche Lage, Organisation und Ausstattung der Praxis berücksichtigt.

\section{Lehrpraxen - Engagement auch in der studentischen Lehre und Forschung}

Als aktives Weiterbildungsprogramm stellt das IHAMZ-PA-Programm auch hohe Anforderungen an die Weiterbildungsqualität und damit an die Weiterbildungsstätten. page des Instituts www.hausarztmedizin.uzh.ch verfügbar ist. Hier sind insbesondere die Qualifikation, die Praxisgegebenheiten und die Zusammenarbeit mit dem Institut darzulegen.

Es sei nochmals betont dass das Praxisassistenzprogramm des IHAMZ wie dargelegt kein Co-Finanzierungsprogramm ist, es ist daher nicht möglich, dass sich Lehrpraktiker mit einem Assistenten zusammen bewerben, um eine Co-Finanzierung zu erhalten. Hierfür stehen andere Programme zur Verfügung.

\section{Ausblick}

Mit dem umfangreichen Praxisassistenzprogramm rundet das IHAMZ eine nahtlose Begleitung und Förderung des potentiellen hausärztlichen Nachwuchses vom ersten Semester bis hin zum letzten Weiterbildungsabschnitt ab. Damit sind fünf Jahre nach Gründung des Institutes in Zürich alle Möglichkeiten, die im Einflussgebiet einer universitären Institution liegen, um den hausärztlichen Nachwuchs zu erhöhen, umgesetzt. hochstehende, an der aktuellen medizinischen Evidenz orientierte Grundversorgung. Sie arbeiten mit dem Institut für Hausarztmedizin der Universität Zürich auch in der Forschung und in der studentischen Lehre (Ausbildung) zusammen und besuchen die dafür vorgesehenen Fortbildungen, wie etwa die Didaktikseminare und die Lehrärztetreffen. Sie führen regelmässige arbeitsplatzbasierte Assessments (ABAS) durch und werden für die Akkreditierung von einem Insti-

\section{Dank}

Die Realisierung des Praxisassistenzprogrammes wäre nicht möglich gewesen ohne die tatkräftige und unkomplizierte Unterstützung vieler Personen und Institutionen. Besonderer Dank gilt hier der Gesundheitsdirektion des Kantons Zürich für den nötigen Rückenwind. Wie bereits beim Hausarzt-Curriculum

\section{Es steht auch Kollegen offen, die sich hinsichtlich ihrer späteren Tätigkeit noch nicht sicher sind und sich in einer hausärztlichen Praxis orientieren wollen.}

tutsmitarbeiter auch persönlich visitiert. Sämtliche Praxen werden zudem durch die Assistenten im Hinblick auf die Weiterbildungsqualität regelmässig evaluiert. Diese Regelungen dienen vor allem der Qualitätssicherung einer hochwertigen Weiterbildung, sie sollen aber auch dem Umstand Rechnung tragen, dass es für das Institut zunehmend schwieriger wird, ausreichend Lehrärzte für die studentische Lehre zu finden. Es erscheint naheliegend, primär jene Ärzte in die Weiterbildung von Assistenten einzubinden, die ihre didaktischen Fähigkeiten bereits in der Ausbildung von Studierenden erfolgreich unter Beweis gestellt haben.

Ärzte, die Praxisassistenten im Rahmen des Curriculums oder der Praxisassistenz aufnehmen möchten, müssen sich beim Institut bewerben. Für die Erfassung der Lehrpraxis ist ein elektronisch ausgefülltes Praxiserfassungsblatt notwendig, das auf der Home- hat sich das UniversitätsSpital Zürich erneut sehr konstruktiv und speditiv in der Realisierung gezeigt. Das USZ nimmt hier auf vorbildliche Weise seine gemeinwirtschaftliche Aufgabe wahr, auch dafür grossen Dank, insbesondere an die Spitaldirektion, namentlich an Frau Rita Ziegler und den Ärztlichen Direktor, Prof. Jürg Hodler.

\section{Literatur}

1 Badertscher N, Schöni M, Berger L, Zoller M, Rosemann T. Vocational training of future GPs in Swiss hospitals: the view of assistant physicians. Praxis. 2011;100(6):349-55.

2 Rosemann T, Bandi-Ott E, Tandjung R. Das Curriculum für Hausarztmedizin am USZ - eine Erfolgsgeschichte. Schweiz Ärztezeitung. 2012;93(39):1432-3. 